\title{
Glue Spin and Helicity in the Proton from Lattice QCD
}

\author{
Yi-Bo Yang, ${ }^{1}$ Raza Sabbir Sufian, ${ }^{1}$ Andrei Alexandru, ${ }^{2}$ Terrence Draper, ${ }^{1}$ Michael J. Glatzmaier, ${ }^{1}$ \\ Keh-Fei Liu, ${ }^{1}$ and Yong Zhao, \\ $(\chi \mathrm{QCD}$ Collaboration)

\begin{abstract}
${ }^{1}$ Department of Physics and Astronomy, University of Kentucky, Lexington, Kentucky 40506, USA ${ }^{2}$ Department of Physics, The George Washington University, Washington, D.C. 20052, USA

${ }^{4}$ Nuclear Science Division, Lawrence Berkeley National Laboratory, Berkeley, California 94720, USA

(Received 12 October 2016; revised manuscript received 6 December 2016; published 6 March 2017)
\end{abstract} \\ ${ }^{3}$ Maryland Center for Fundamental Physics, University of Maryland, College Park, Maryland 20742, USA
}

\begin{abstract}
We report the first lattice QCD calculation of the glue spin in the nucleon. The lattice calculation is carried out with valence overlap fermions on $2+1$ flavor domain-wall fermion gauge configurations on four lattice spacings and four volumes including an ensemble with physical values for the quark masses. The glue spin $S_{G}$ in the Coulomb gauge in the modified minimal subtraction $(\overline{\mathrm{MS}})$ scheme is obtained with one-loop perturbative matching. We find the results fairly insensitive to lattice spacing and quark masses. We also find that the proton momentum dependence of $S_{G}$ in the range $0 \leq|\vec{p}|<1.5 \mathrm{GeV}$ is very mild, and we determine it in the large-momentum limit to be $S_{G}=0.251(47)(16)$ at the physical pion mass in the $\overline{\mathrm{MS}}$ scheme at $\mu^{2}=10 \mathrm{GeV}^{2}$. If the matching procedure in large-momentum effective theory is neglected, $S_{G}$ is equal to the glue helicity measured in high-energy scattering experiments.
\end{abstract}

DOI: 10.1103/PhysRevLett.118.102001

Introduction.-Deep-inelastic scattering experiments reveal that, contrary to the naive quark model, the quark spin contribution to the proton spin is quite small, about $30 \%$ [1-3]. In an effort to search for the missing proton spin, recent analyses [4,5] of the high-statistics 2009 STAR [6] and PHENIX [7] experiments at RHIC showed evidence of nonzero glue helicity $\Delta G$ in the proton. For $Q^{2}=10 \mathrm{GeV}^{2}$, the glue helicity distribution $\Delta g\left(x, Q^{2}\right)$ is found to be positive and away from zero in the momentum fraction region $x<0.05$. However, the results are limited by very large uncertainty in this region.

The recent COMPASS analysis explored $\Delta g(x)$ from the scaling violation of $\Delta q(x)$, and the highly distinct solutions of $\Delta g(x)$ can be obtained with different parametrizations of $\Delta q(x)$ [8]. Therefore, it hints that if a high precision $\Delta g(x)$ can be obtained directly, it will benefit our understanding of the parametrizations of $\Delta q(x)$ and provide more information about the role of quark spin in the proton.

Given the importance of $\Delta g(x)$ to explain the origin of the proton spin, and the fact that significant efforts are devoted to its precise experimental determination, a theoretical understanding and calculation of $\Delta G$ is highly desired. $\Delta G$ is defined as the first moment of the glue helicity distribution $\Delta g(x)$ [9],

$$
\begin{aligned}
\Delta G= & \int d x \frac{i}{2 x P^{+}} \int \frac{d \xi^{-}}{2 \pi} e^{-i x P^{+} \xi^{-}} \\
& \times\left\langle P S\left|F_{a}^{+\alpha}\left(\xi^{-}\right) \mathcal{L}^{a b}\left(\xi^{-}, 0\right) \tilde{F}_{\alpha, b}^{+}(0)\right| P S\right\rangle,
\end{aligned}
$$

where the light front coordinates are $\xi^{ \pm}=\left(\xi^{0} \pm \xi^{3}\right) / \sqrt{2}$. The proton plane wave state is written as $|P S\rangle$, with momentum $P^{\mu}=(P, 0,0, P)$ and polarization $S$. The lightcone gauge-link $\mathcal{L}\left(\xi^{-}, 0\right)=P \exp \left[-i g \int_{0}^{\xi-} A^{+}\left(\eta^{-}, 0_{\perp}\right) d \eta^{-}\right]$ is defined in the adjoint representation. It connects the gauge field tensor and its dual, $\tilde{F}^{\alpha \beta}=\frac{1}{2} \epsilon^{\alpha \beta \mu \nu} F_{\mu \nu}$, to construct a gauge-invariant operator. After integrating over $x$, one can define the gauge-invariant gluon helicity operator in a nonlocal form $[10,11]$,

$\tilde{S}_{g}=\left[\vec{E}^{a}(0) \times\left(\vec{A}^{a}(0)-\frac{1}{\nabla^{+}}\left(\vec{\nabla} A^{+, b}\right) \mathcal{L}^{b a}\left(\xi^{-}, 0\right)\right)\right]^{z}$,

where $\nabla^{+}=\partial / \partial \xi^{-}$. It is the gauge-invariant extension of the operator $\vec{E} \times \vec{A}$ in the light-cone gauge $A^{+}=0$, but one cannot evaluate this expression on the lattice directly due to its real-time dependence.

On the other hand, $\tilde{S}_{g}$ is equal to the infinite momentum frame (IMF) limit of a universality class of operators [12] whose matrix elements can be matched to $\Delta G$ through a factorization formula in large-momentum effective theory (LMET) [13,14]. The gluon spin operator proposed in Ref. [15,16] with the non-Abelian transverse condition belongs to this universality class and has been proven to be equivalent to the gauge-invariant extension of $\vec{E} \times \vec{A}$ in the Coulomb gauge $\vec{\partial} \cdot \vec{A}=0[17,18]$, 


$$
\vec{S}_{g}=2 \int d^{3} x \operatorname{Tr}\left(\vec{E}_{c} \times \vec{A}_{c}\right),
$$

where the factor 2 is from the normalization of the SU(3) group generators and $\vec{E}_{c}$ and $\vec{A}_{c}$ are the chromoelectric field and gauge potential in the Coulomb gauge with their lattice versions to be addressed in the following.

$\vec{S}_{g}$ is not Lorentz covariant and has nontrivial frame dependence [11]. It is shown in Ref. [12] that when boosted to the IMF, the Coulomb gauge fixing condition (as well as the temporal condition $A^{0}=0$ ) [12] becomes $A^{+}=0$, and then the longitudinal component of $\vec{S}_{g}$ in either gauge is equivalent to the glue helicity operator $\tilde{S}_{g}$ with a proper matching to cancel the intrinsic frame dependence of $\vec{S}_{g}$. On the lattice, the Coulomb condition can be obtained numerically [19] and the glue spin operator $\vec{S}_{g}$ in the Coulomb gauge can be calculated without numerical difficulty.

The major task of this work is calculating the matrix element of $\vec{S}_{g}$ in the proton, which will be indicated as $S_{G}$, in the rest and moving frames. The results are then renormalized at one-loop order in lattice perturbation theory and matched to the modified minimal subtraction ( $\overline{\mathrm{MS}}$ ) scheme at $\mu^{2}=10 \mathrm{GeV}^{2}$, to investigate their frame dependence and address the matching to the helicity.

Numerical details. - A preliminary attempt [20] to calculate $S_{G}$ was carried out on $2+1$ flavor dynamical domain-wall configurations on a $24^{3} \times 64$ lattice (24I) with the sea pion mass at $330 \mathrm{MeV}$ and on a $32^{3} \times 64$ lattice with sea pion mass at $300 \mathrm{MeV}$ [21]. In this work, we improve the statistics on the ensembles mentioned above and carry out the calculation on another three ensembles with different lattice spacings, volumes, and sea quark masses to check the corrections to the glue spin from various systematic uncertainties. We use the 2-2-2 smeared stochastic grid source on all the ensembles (except 48I, where the 4-4-4 smeared stochastic grid source is used), and apply the low-mode substitution [22,23] to make the signal-to-noise ratio close to that with 8 (64 on the 48I ensemble) independent smeared point sources. Furthermore, we loop over all the time slices for the two-point functions of the nucleon to increase statistics. The statistics used for this grid source measurement is roughly equivalent to evaluating a large number of quasiindependent smeared point source measurements ranging from 103,936 on the 24I lattice to 497,664 on the $48 \mathrm{I}$ lattice. The parameters of the ensembles used in this work are listed in Table I, and more details of the simulation setups can be found in the Supplemental Material [24].

The Coulomb gauge fixing condition used here is enforced by requiring that the spatial sum of the backward difference of the hypercubic (HYP)-smeared gauge links [26] be zero,
TABLE I. The parameters for the RBC and UKQCD configurations [25]. $m_{\pi}^{(s)}$ is the pion mass of the light sea quark in the $2+1$ flavor configuration, and $N_{\text {cfg }}$ is the number of configurations used in the simulation.

\begin{tabular}{lcccr}
\hline \hline Symbol & $L^{3} \times T$ & $a(\mathrm{fm})$ & $m_{\pi}^{(s)}(\mathrm{MeV})$ & $N_{\text {cfg }}$ \\
\hline 32ID & $32^{3} \times 64$ & $0.1431(7)$ & 170 & 200 \\
48I & $48^{3} \times 96$ & $0.1141(2)$ & 140 & 81 \\
24I & $24^{3} \times 64$ & $0.1105(3)$ & 330 & 203 \\
32I & $32^{3} \times 64$ & $0.0828(3)$ & 300 & 309 \\
32If & $32^{3} \times 64$ & $0.0627(3)$ & 370 & 238 \\
\hline \hline
\end{tabular}

$$
\sum_{\mu=x, y, z}\left[U_{\mu}^{c}(x)-U_{\mu}^{c}(x-a \hat{\mu})\right]=0,
$$

where $U_{\mu}^{c}(x)$ is the Coulomb gauge fixed Wilson link from $x+a \hat{\mu}$ to $x$. The gauge fixed potential $A_{c}$ is defined by

$A_{c, \mu}=\left(\frac{U_{\mu}^{c}(x)-U_{\mu}^{c \dagger}(x)+U_{\mu}^{c}(x-a \hat{\mu})-U_{\mu}^{c \dagger}(x-a \hat{\mu})}{4 i a g}\right)_{\text {traceless }}$,

with $g$ as the bare coupling constant, and the chromoelectric field used in this work is given by the clover definition,

$$
\begin{aligned}
F_{\mu \nu}^{c}= & \frac{i}{8 a^{2} g}\left(\mathcal{P}_{\mu, \nu}-\mathcal{P}_{\nu, \mu}+\mathcal{P}_{\nu,-\mu}-\mathcal{P}_{-\mu, \nu}\right. \\
& \left.+\mathcal{P}_{-\mu,-\nu}-\mathcal{P}_{-\nu,-\mu}+\mathcal{P}_{-\nu, \mu}-\mathcal{P}_{\mu,-\nu}\right),
\end{aligned}
$$

where $\mathcal{P}_{\mu, \nu}=U_{\mu}^{c}(x) U_{\nu}^{c}(x+a \hat{\mu}) U_{\mu}^{c \dagger}(x+a \hat{\nu}) U_{\nu}^{c \dagger}(x)$.

In order to extract $S_{G}$, we compute the ratio of the disconnected three-point function with the gluon operator insertion to the nucleon propagator with the source and sink of the nucleon located at 0 and $t_{f}$, respectively. The glue spin operator is inserted at the time slice $t$, which is between 0 and $t_{f}$. Then the ratio in a moving frame $\vec{p}=\left(0,0, p_{3}\right)$ along the $z$ direction is

$R\left(t_{f}, t\right)=\frac{\left\langle 0\left|\Gamma_{3}^{m} \int d^{3} y e^{-i p_{3} y_{3}} \chi\left(\vec{y}, t_{f}\right) S_{g}^{3}(t) \bar{\chi}(\overrightarrow{0}, 0)\right| 0\right\rangle}{\left\langle 0\left|\Gamma^{e} \int d^{3} y e^{-i p_{3} y_{3}} \chi\left(\vec{y}, t_{f}\right) \bar{\chi}(\overrightarrow{0}, 0)\right| 0\right\rangle}$,

where $\chi$ is the nucleon interpolation field and $\Gamma^{e}$ and $\Gamma_{3}^{m}$ are the unpolarized projection operator of the proton and the polarized one along the $z$ direction, respectively. When $t_{f}$ is large enough, $R\left(t_{f}, t\right)$ is equal to the proton matrix element of the longitudinal glue spin operator $S_{G}$ plus $t$-dependent corrections,

$R\left(t_{f}, t\right)=S_{G}+C_{1} e^{-\Delta E\left(t_{f}-t\right)}+C_{2} e^{-\Delta E t}+C_{3} e^{-\Delta E t_{f}}$,

where $\Delta E$ is the energy difference between the first excited state and the ground state and $C_{1,2,3}$ are the spectral weights involving the excited state. 


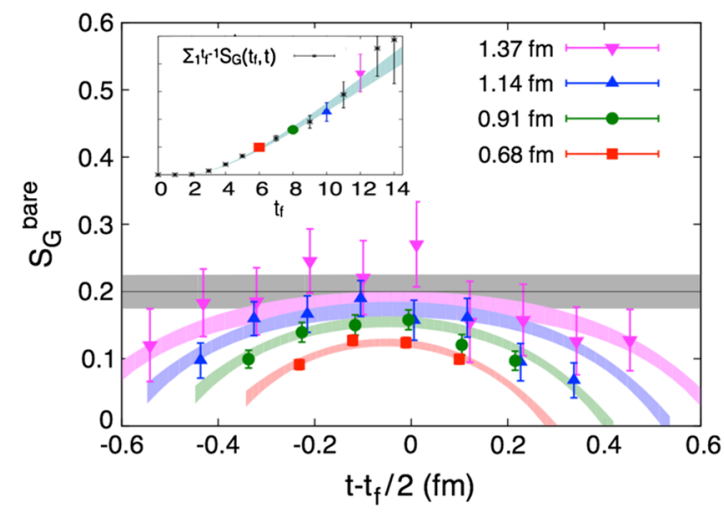

FIG. 1. The ratio $R\left(t_{f}, t\right)$ as a function of the source-sink separation $t_{f}$ and the current time slice $t$, for the bare glue spin matrix element in the proton $S_{G}^{\text {bare }}$ is plotted at the unitary point on the 24I ensemble. The gray band shows the result extrapolated to infinite separation, which corresponds to the prediction of $S_{G}$. The excited-state contamination is small when the source-sink separation is larger than $1 \mathrm{fm}$. The inset shows that the prediction of $\sum_{t} R\left(t_{f}, t\right)$ from the two-state fit (the band) in Eq. (8) agrees well with the data points.

We plot the ratio $R\left(t_{f}, t\right)$ for the unitary point on the 24I ensemble, as a function of $t-t_{f} / 2$ for several $t_{f}$, in Fig. 1. The curves predicted by the fit agree with the data, and the $\chi^{2} /$ d.o.f. is smaller than 1.4 for all the other quark masses on five ensembles. From the fit, we see that the excitedstate contamination is small when the source-sink separation is larger than $1 \mathrm{fm}$. The final prediction of $S_{G}$ (the gray band) is consistent with the blue and purple data points at $t \sim t_{f} / 2$. Similar plots for the other ensembles can be found in the Supplemental Material [24].

It is observed that the central values of the glue spin matrix elements as a function of HYP-smearing steps are unchanged after two or three steps of smearing, as shown in Fig. 2 (for the case of the unitary point on the ensemble

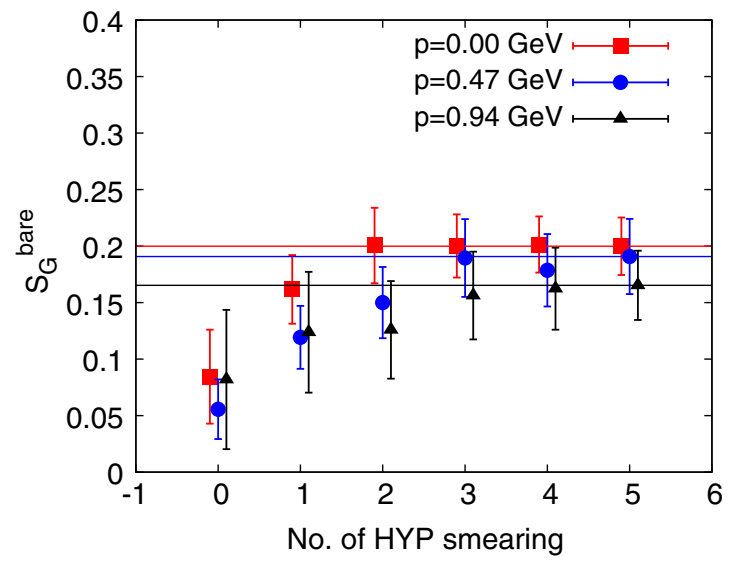

FIG. 2. The HYP-smearing steps dependence of the bare glue spin $S_{G}$ at the unitary point on the ensemble 24I, for $p=0$ (red squares), $p=0.47 \mathrm{GeV}$ (blue dots), and $p=0.94 \mathrm{GeV}$ (black triangles). The values of $S_{G}$ are unchanged after two or three steps of smearing.
24I), while the SNR can be improved when more HYP-smearing steps are applied. In this work, five steps of HYP smearing are used for the glue spin operator on each ensemble, and the nucleon two-point correlators with the source located on all the time slices are generated to increase the SNR. Since the tadpole improvement factor is $1 / u_{0}^{5} \sim 2$ for the $S_{g}$ operator without any HYP smearing, the enlargement of the result after the HYP smearing is understandable. Note that the HYP smearing here just affects the glue spin operator, but the gauge action is unchanged since no reweighting is applied on configuration averages.

Results.-The renormalized matrix element $S_{G}$ including mixing from the quark spin is [27]

$$
\begin{aligned}
S_{G}^{\mathrm{MS}}= & \left\{1-\frac{g^{2}}{16 \pi^{2}}\left[N_{f}\left(\frac{2}{3} \log \left(\mu^{2} a^{2}\right)+1.27\right)\right.\right. \\
& \left.\left.-C_{A}\left(\frac{4}{3} \log \left(\mu^{2} a^{2}\right)+f_{g g}\left(g^{2}\right)\right)\right]\right\} S_{G}^{L} \\
& +\frac{g^{2} C_{F}}{16 \pi^{2}}\left(\frac{5}{3} \log \left(\mu^{2} a^{2}\right)+7.31\right) \Delta \Sigma^{L}+O\left(g^{4}\right),
\end{aligned}
$$

where the superscripts $\overline{\mathrm{MS}}$ and $L$ indicate the quantities under the $\overline{\mathrm{MS}}$ scheme and that under lattice regularization, respectively. We applied the cactus improvement [28] to resum the major tadpole contributions to get a better convergence in the one-loop correction of the glue spin. Then the resummed finite piece $f_{g g}\left(g^{2}\right)$ depends on the bare coupling $g^{2}$ weakly and is in the range of 1.7-2.4 for the values of $g^{2}$ we used in this work. The details are addressed in Ref. [27]. Since the value of the mixing term involving $\Delta \Sigma^{L}$ in Eq. (9) is at the same order of the present statistical error of $S_{G}^{L}$, the uncertainty due to $\Delta \Sigma^{L}$ for the gauge ensembles considered here will be even smaller. Therefore, we approximate the quark spin $\Delta \Sigma^{L}$ by the experimental value $\Delta \Sigma^{\mathrm{MS}}$, which is $\sim 30 \%$ of the total proton spin from the global analysis of deep-inelastic scattering data [1-3].

After matching to the values under the $\overline{\mathrm{MS}}$ scheme at $\mu^{2}=10 \mathrm{GeV}^{2}$, we find that the valence quark mass dependence is mild regardless of the proton momentum. In Fig. 3, we show results in the rest frame for various valence quark masses on all the five ensembles, with five pairs of volumes and lattice spacings. Their dependence is also mild. To obtain $S_{G}$ in a relatively large-momentum frame, we calculate $S_{G}$ for all the momenta smaller than $\pi /(4 a)$ on all the five ensembles. To show the frame dependence, we extrapolate $S_{G}$ on all the ensembles in different momentum frames to the physical value of the valence pion mass, as shown in Fig. 4. Some points are not shown in the figure if their uncertainties are larger than the signal. 


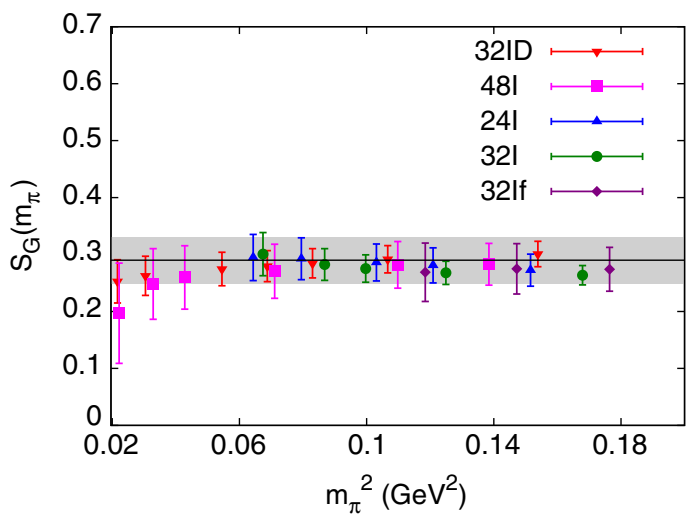

FIG. 3. The valence pion mass dependence of $S_{G}$ at $\mu^{2}=10 \mathrm{GeV}^{2}$, in the rest frame of the proton. These dependencies are fairly mild and can be well described with a linear fit. The gray band shows the result based on the global fit with the empirical form in Eq. (11).

The glue helicity in the proton $\Delta G$ corresponds to the glue longitudinal spin component $S_{G}$ in the IMF. The LMET [14] shows a large finite correction at the one-loop level:

$$
\begin{aligned}
S_{G}(|\vec{p}|, \mu)= & {\left[1+\frac{g^{2} C_{A}}{16 \pi^{2}}\left(\frac{7}{3} \log \frac{(\vec{p})^{2}}{\mu^{2}}-10.2098\right)\right] \Delta G(\mu) } \\
& +\frac{g^{2} C_{F}}{16 \pi^{2}}\left(\frac{4}{3} \log \frac{(\vec{p})^{2}}{\mu^{2}}-5.2627\right) \Delta \Sigma(\mu) \\
& +O\left(g^{4}\right)+O\left(\frac{1}{(\vec{p})^{2}}\right) .
\end{aligned}
$$

At $\mu^{2}=10 \mathrm{GeV}^{2}$ and $|\vec{p}|=1.5 \mathrm{GeV}$, the factor before $\Delta G$ is 0.22 , which is much smaller than unity and indicates a convergence problem for the perturbative series even after one resums the large logarithms. (The factor is 0.80 if the finite piece 10.2098 is removed.) On the other hand, the largest momentum we have on the lattice with acceptable signal is comparable to the proton mass, so the power corrections in Eq. (10) cannot be neglected and one cannot simply apply this matching condition. Nevertheless, the mild dependence of $S_{G}$ on the proton momentum as in Fig. 4 leads us to suggest that it could be a small effect to match to the IMF; i.e., $S_{G} \approx \Delta G+O\left(1 /(\vec{p})^{2}\right)$.

Therefore, we neglect the one-loop LMET matching and use the following empirical form to fit our data:

$$
\begin{aligned}
S_{G}(|\vec{p}|)= & S_{G}(\infty)+\frac{C_{1}}{M^{2}+(\vec{p})^{2}}+C_{2}\left(m_{\pi, v v}^{2}-m_{\pi, \text { phys }}^{2}\right) \\
& +C_{3}\left(m_{\pi, s s}^{2}-m_{\pi, \text { phys }}^{2}\right)+C_{4} a^{2}
\end{aligned}
$$

where $m_{\pi \text {,phys }}=0.139 \mathrm{GeV}$ and $M=0.939 \mathrm{GeV}$ are the physical pion and proton mass, respectively, and $m_{\pi, v v / s s}$ are the valence and sea pion masses, respectively. The

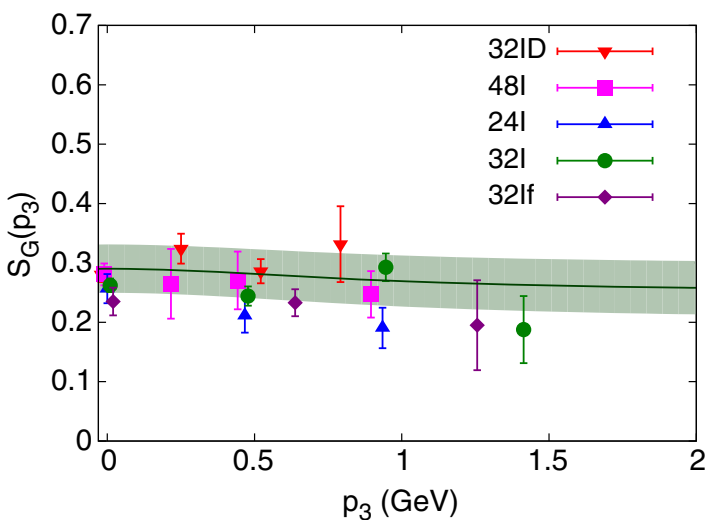

FIG. 4. The results extrapolated to the physical pion mass as a function of the absolute value of $\vec{p}=\left(0,0, p_{3}\right)$, on all the five ensembles. All the results have been converted to $\overline{\mathrm{MS}}$ at $\mu^{2}=10 \mathrm{GeV}^{2}$. The data on several ensembles are shifted horizontally to enhance the legibility. The green band shows the frame dependence of the global fit [with the empirical form in Eq. (11)] of the results.

$1 /(\vec{p})^{2}$ correction in Eq. (10) is replaced by $1 /\left[M^{2}+(\vec{p})^{2}\right]$ to include all the data in the fitting. Since all the coefficients other than $S_{G}(\infty)$ are small, the cross terms and the higherorder terms are ignored. The overall $\chi^{2} /$ d.o.f. is 1.21 with 110 degrees of freedom. In Fig. 4, the band of the global fit with the empirical form in Eq. (11) shows that the frame dependence is mild and the central value is changed by less than $10 \%$ from its value in the rest frame to that at $|\vec{p}| \sim 1.5 \mathrm{GeV}$; the change is smaller than the statistical uncertainty.

Since the Coulomb gauge fixing on the lattice has a builtin $O(a)$ correction, we repeated the fit with a linear term in $a$. The central value is changed by about $1 \%$, while the uncertainty is larger. We take the variance of the central values from two fits as an estimate of this uncertainty. Similarly, the uncertainty from the volume dependence $e^{-m_{\pi_{v v}} L}$ is estimated in the same way and added to the systematic uncertainties in quadrature. In addition, the value of the quark spin $\Delta \Sigma$ is varied by $20 \%$ to cover the value $\sim 0.30$ [1] and that from Ref. [3]. The final result is $S_{G}\left(\infty, \mu^{2}=10 \mathrm{GeV}^{2}\right)=0.251(47)(16)$ with two errors from the statistical and systematic uncertainties.

Summary and outlook.-In this work, we calculated the glue spin in the proton for the first time based on $\vec{E} \times \vec{A}$ in the Coulomb gauge $[15,16]$, with various quark masses, lattice spacings, volumes, and proton momenta. The results show mild dependencies on these quantities. After one-loop perturbative matching from the lattice theory to the continuum and neglecting the matching effect between the glue spin and helicity, we conclude that the gluon helicity $\Delta G\left(\mu^{2}=10 \mathrm{GeV}^{2}\right) \approx S_{G}\left(\infty, \mu^{2}=10 \mathrm{GeV}^{2}\right)=0.251(47)(16)$, which is 50(9)(3)\% of the total proton spin. The cactus improvement [28] we used in Eq. (9) indicates that uncertainties can be considerable in perturbative QCD, 
and its reliability should be checked with nonperturbative renormalization in the future.

On the LMET side, the convergence problem warrants the matching condition to be calculated at the two-loop level or higher. On the other hand, if the glue spin in the temporal gauge can be calculated on the lattice, then its LMET matching in Eq. (10) can be avoided at the oneloop level [12]. This possibility is worthy of further investigation [27].

We thank X. D. Ji and F. Yuan for useful comments and the RBC and UKQCD Collaborations for providing us their domain-wall fermion gauge configurations. This work is supported in part by the U.S. DOE Grant No. DESC0013065. A. A. is supported in part by the National Science Foundation CAREER Grant No. PHY-1151648 and by U.S. DOE Grant No. DE-FG02-95ER40907. Y.Z. is supported in part by the U.S. Department of Energy Office of Science, Office of Nuclear Physics under Awards No. DE-FG02-93ER-40762 and No. DE-AC0205CH11231. Y.-B. Y. also thanks the Institute of High Energy Physics, Chinese Academy of Science for its partial support and hospitality. This material is based upon work supported by the U.S. Department of Energy, Office of Science, Office of Nuclear Physics, within the framework of the TMD Topical Collaboration. This research used resources of the Oak Ridge Leadership Computing Facility at the Oak Ridge National Laboratory, which is supported by the Office of Science of the U.S. Department of Energy under Contract No. DE-AC05-00OR22725. This work also used the Extreme Science and Engineering Discovery Environment (XSEDE), which is supported by National Science Foundation Grant No. ACI-1053575.

[1] A. Accardi et al., Eur. Phys. J. A 52, 268 (2016).

[2] D. de Florian, R. Sassot, M. Stratmann, and W. Vogelsang, Phys. Rev. D 80, 034030 (2009).

[3] C. A. Aidala, S. D. Bass, D. Hasch, and G. K. Mallot, Rev. Mod. Phys. 85, 655 (2013).

[4] D. de Florian, R. Sassot, M. Stratmann, and W. Vogelsang, Phys. Rev. Lett. 113, 012001 (2014).
[5] E. R. Nocera, R. D. Ball, S. Forte, G. Ridolfi, and J. Rojo (NNPDF Collaboration), Nucl. Phys. B887, 276 (2014).

[6] P. Djawotho (STAR Collaboratio), Nuovo Cimento Soc. Ital. Fis. 36C, 35 (2013).

[7] A. Adare et al. (PHENIX Collaboration), Phys. Rev. D 90, 012007 (2014).

[8] C. Adolph et al. (COMPASS Collaboration), Phys. Lett. B 753, 18 (2016).

[9] A. V. Manohar, Phys. Lett. B 255, 579 (1991).

[10] Y. Hatta, Phys. Rev. D 84, 041701 (2011).

[11] X. Ji, J.-H. Zhang, and Y. Zhao, Phys. Rev. Lett. 111, 112002 (2013).

[12] Y. Hatta, X. Ji, and Y. Zhao, Phys. Rev. D 89, 085030 (2014).

[13] X. Ji, Sci. China Phys. Mech. Astron. 57, 1407 (2014).

[14] X. Ji, J.-H. Zhang, and Y. Zhao, Phys. Lett. B 743, 180 (2015).

[15] X.-S. Chen, X.-F. Lu, W.-M. Sun, F. Wang, and T. Goldman, Phys. Rev. Lett. 100, 232002 (2008).

[16] X.-S. Chen, W.-M. Sun, X.-F. Lu, F. Wang, and T. Goldman, Phys. Rev. Lett. 103, 062001 (2009).

[17] C. Lorce, Phys. Rev. D 87, 034031 (2013).

[18] Y. Zhao, K.-F. Liu, and Y. B. Yang, Phys. Rev. D 93, 054006 (2016).

[19] M. Schröck and H. Vogt, Comput. Phys. Commun. 184, 1907 (2013).

[20] R. S. Sufian, M. J. Glatzmaier, Y.-B. Yang, K.-F. Liu, and M. Sun ( $\chi$ QCD), Proc. Sci., LATTICE2014 (2014) 166.

[21] Y.-B. Yang, R. S. Sufian, A. Alexandru, T. Draper, M. J. Glatzmaier, and K.-F. Liu ( $\chi$ QCD Collaboration), Proc. Sci., LATTICE (2015) 129.

[22] A. Li et al. ( $\chi$ QCD Collaboration), Phys. Rev. D 82, 114501 (2010).

[23] M. Gong et al. ( $\chi$ QCD Collaboration), Phys. Rev. D 88, 014503 (2013).

[24] See Supplemental Material at http://link.aps.org/ supplemental/10.1103/PhysRevLett.118.102001 for the details of the numerical simulation setup and the two-state fits used to obtain the glue spin matrix element.

[25] T. Blum et al. (RBC and UKQCD Collaborations), Phys. Rev. D 93, 074505 (2016).

[26] A. Hasenfratz and F. Knechtli, Phys. Rev. D 64, 034504 (2001).

[27] Y.-B. Yang and Y. Zhao (to be published).

[28] M. Constantinou, H. Panagopoulos, and A. Skouroupathis, Phys. Rev. D 74, 074503 (2006). 